\title{
Segmentation of Medical Images using Adaptively Regularized Kernel-based Fuzzy C-Means Clustering
}

\author{
Neha Tomar \\ M.Tech Scholar \\ Dept. of C.S.E \\ Sachdeva Institute of Technology \\ Mathura
}

\author{
Vyom Kulshreshtha \\ Assistant Prof. \\ Dept. of C.S.E \\ Sachdeva Institute of Technology \\ Mathura
}

\author{
Pankaj Sharma \\ H.O.D \\ Dept. of C.S.E \\ Sachdeva Institute of Technology \\ Mathura
}

\begin{abstract}
Medical imaging is the process of seeing the internal parts of the body, whose purpose is to maintain health, prevention and treatment of diseases. Nowadays, medical imaging has become a common part of everyday clinical practices. Despite huge progress, there is still no such instrument that can represent all facets of the human body. Image segmentation is the most common method used to analyze and detect distortion in medical images. Clustering is a technique used to group similar data in the same cluster. MRI segmentation is critically important for diagnostic studies and diagnosis. There are many drawbacks in existing methods based on soft clustering, which include low noise and high computational cost in the presence of image noise and artifacts. In this paper, we use a novel method to split brain tissues from magnetic resonance images, which routinely use regularized kernel-based fuzzy-clustering clusters. Adaptive regularized kernel based fuzzy clustering means (ARKFCM) is applied to remove nuclei and non-nuclei images of the Histopathological ROI image. In ARKFCM, results occurred in two sets of images, both images are examined to generate clustering cells. The combined ARKFCM image contains many overlapping areas of cells.
\end{abstract}

\section{Keywords}

Image segmentation; MRI; k-means; FCM; ARKFCM;

\section{INTRODUCTION}

A brain tumor or intracranial neoplasm occurs when abnormal cells form within the brain. There are several ways to diagnose brain tumors, for example use MRI images. Nowadays, Medical imaging plays a significant role in everyday clinical use. There are a number of different imaging modalities that each shows specific aspect of the human body. Each of modalities such as magnetic resonance imaging (MRI), computed tomography (CT), etc., provides limited and complementary information. As an instance, CT can indicate bones and dense tissue with great details. However, it provides little information about soft tissue, in contrast to MRI which displays soft tissue with high resolution.[1]

In the field of biomedical imaging and computer-aided diagnosis, image acquisition and analysis with more than one modality (i.e. multi-modal) has been a research focus for years since different imaging modalities encompass abundant information which are complementary to each other. As described in one of the multi-modal imaging project for brain tumor segmentation, each of the modality can reveal a unique type of biological/biochemical information for the tumorinduced tissue changes and poses. Somewhat different information processing tasks.[2]. The segmentation of biomedical images into the various tissues and structures forms a crucial step for both medical research and clinical practice.
Automatic segmentation is important because manually segmenting three-dimensional images is very time consuming and prone to inter- and inter observer variability.[3]

Clustering is a method of grouping similar data and distinctly separating them from the dissimilar data. It helps recognizing hidden patterns within the data. It is an unsupervised approach. For pattern extraction, clustering techniques depend on the similarity measures between the representative and the data to be clustered. Representative data denotes the cluster center, i.e., the ideal data of the cluster. Clustering is a useful tool for understanding and visualizing available structures in data. Fuzzy C-means is one of the commonly used and efficient objective function-which is based on clustering techniques. Data clustering or cluster analysis is an important field in pattern recognition, machine intelligence and computer vision community, that has had numerous applications in the last three decades. Generally, clustering term is known as grouping a set of $\mathrm{N}$ samples into $\mathrm{C}$ clusters whose members are similar in some sense. This similarity between different samples is either a suitable distance based on numeric attributes, or directly in the form of pairwise similarity or dissimilarity measurements. With a clustering technique, a collection of objects or feature vectors is partitioned into clusters. In the past few decades, many clustering algorithms have been developed, which mainly contain hierarchical clustering (such as Single Link and Complete Link), partitional clustering (such as $\mathrm{k}$ means, fuzzy C-means, Gaussian Mixture and Density Estimation) and spectral clustering. Moreover, in the last few years, fuzzy Cmeans (FCM) clustering and spectral clustering algorithms are research focus.[4]

Fuzzy clustering introduces the concept of membership into data partition, for this reason that membership can indicate the degree to which an object belongs to the clusters definitely, and actually represents the data partition more clearly.[5]. Study of cluster or segmentation technique [9], [10] based on clustering assembles a set of entities in a manner that entities in the identical cluster have a superior degree of alikeness to each compared to the other clusters. Clusters are defined as contiguous regions of more than one-dimensional space comprising comparative points of high density, alienated from other exemplary regions comprising moderate points of low density. In image breakdown, clustering is the order of arrangement of pixels conferring to more or less features like intensity. Under hard clustering, data elements fit into one cluster simply and the membership value of belongingness to a cluster is precisely one. Under soft clustering, elements of data fit into more prominent than the single cluster and the membership value of belongingness to the cluster varies from 0 to $1[6]$.

This paper is structured as follows. In section II, papers which deal with similar technique description. Section III presents 
literature survey of the previous scheme, Section IV presents propose work. Section V presents experiment result analysis and section VII conclusions are drawn.

\section{USING TECHNIQUES}

In this paper we have used two techniques which are described below:

\subsection{K-means (KM) Clustering Algorithm:}

Currently the clustering method often used for segmenting largescale images. Clustering is one of the unsupervised learning method in which a set of essentials is separated into uniform groups. There are different types of clustering: hierarchical clustering, Fuzzy C-means clustering, K-means clustering. The $\mathrm{K}$-means method is one of the most generally used clustering techniques for various applications. K-means clustering is a partition-based cluster analysis method. The K-means clustering technique is a widely used approach that has been applied to solve low-level image segmentation tasks.[7]

Clustering is a method to divide a set of data into a specific number of groups. It's one of the popular method is k-means clustering. In k-means clustering, it partitions a collection of data into a $\mathrm{k}$ number group of data [8] . It classifies a given set of data into $\mathrm{k}$ number of disjoint cluster. K-means algorithm consists of two separate phases. In the first phase it calculates the $\mathrm{k}$ centroid and in the second phase it takes each point to the cluster which has nearest centroid from the respective data point. There are different methods to define the distance of the nearest centroid and one of the most used methods is Euclidean distance. Once the grouping is done it recalculate the new centroid of each cluster and based on that centroid, a new Euclidean distance is calculated between each center and each data point and assigns the points in the cluster which have minimum Euclidean distance. Each cluster in the partition is defined by its member objects and by its centroid. The centroid for each cluster is the point to which the sum of distances from all the objects in that cluster is minimized. So K-means is an iterative algorithm in which it minimizes the sum of distances from each object to its cluster centroid, over all clusters.[8]

\subsection{Fuzzy C-means (FCM) Clustering:}

Fuzzy-C-Means is a soft clustering approach. In the case of soft clustering which is also called Fuzzy clustering, data items can belong to more than one cluster. Membership levels indicate the strength of association between that element and a particular cluster. Basic idea in this algorithm is that the data points have their membership values with the cluster centres that will be iteratively updated. It involves two main steps: the calculation of cluster centers and the assignment of points to these centers. A membership value is assigned to the data items for the cluster within the range of 0 to 1 and a Fuzzification parameter in the range $[1, n]$, to determine the degree of Fuzziness. The following objective function has to be minimized.

$$
\sum_{j=,}^{c} \mu j k=1, \forall K=1, \ldots n
$$

Membership $\mu \mathrm{jk}$ is given by:

$$
\mu j k=\frac{1}{\sum_{p=1}^{c}\left(\frac{\|x j-c k\|}{\|x j-c p\|}\right)^{\frac{2}{m-1}}}
$$

Soft Clustering generate good cluster centroid for optimal solution [9]. FCM Algorithm consists of steps as given below:

1. The membership matrix $U$ is initialized with random values between 0 and 1 such that the constraints are satisfied.
2. Calculate fuzzy cluster centres $\mathrm{ck}$, where $\mathrm{k}=1, \ldots, \mathrm{C}$.

3. Calculate objective function and stop if either it is below a certain tolerance value or its improvement over previous iteration is below a certain threshold.

4. Compute a new membership matrix $U$.

5. Go to step 2 .

6. This iteration will stop if

$\|\mathrm{U}(\mathrm{K}+1)-\mathrm{U}(\mathrm{K})\|<\varepsilon$

Where $\varepsilon$ is the stopping criterion between 0 and 1 where as $\mathrm{K}$ is the iteration steps.[9]

\subsection{Adaptive regularized kernel based fuzzy clustering means (ARKFCM):}

An adaptive kernel-based fuzzy $\mathrm{C}$-means clustering with spatia constraints (AKFCMS) model for image segmentation approach is proposed in order to improve the efficiency of image segmentation. Various experiment results show that the proposed approach can get the spatial information features of an image accurately and is robust to realize image segmentation. An adaptively regularized kernel-based fuzzy $C$-means clustering framework is proposed for segmentation of brain magnetic resonance images. The framework can be in the form of three algorithms for the local average grayscale being replaced by the grayscale of the average filter, median filter, and devised weighted images, respectively. [10]

Initialize threshold $\varepsilon=0.001, m=2$, loop counter $t=0, \mathrm{~V}$, and $u(0)$.

Calculate the adaptive regularization parameter $\varphi i$

Calculate $x i$ for ARKFCM1 and ARKFCM2 or $\xi$ for ARKFCM $w$.

Calculate cluster centers $\mathrm{V}(t) j$ using $u(t)$

Calculate the membership function $(t+1)$.

If $\max \|(t+1)-u(t)\| 100$ then stop; otherwise, update $t=t+1$ and go to step (4).

1. The main advantages are:

a. Adaptive to local context,

b. Enhanced robustness to preserve image details.

c. Independent of clustering parameters, and with decreased computational costs.[10]

\section{LITERATURE REVIEW}

Gijs van Tulder and Marleen de Bruijne, (2018) This paper presents experiments on two public datasets, with knee images from two MRI modalities, provided by the Osteoarthritis Initiative, and brain tumor segmentation on four MRI modalities from the BRATS challenge. All three approaches improved the cross-modality classification accuracy, with modality dropout and per-feature normalization giving the largest improvement. We observed that the networks tend to learn a combination of cross-modality and modality-specific features. Overall, a combination of all three methods produced the most crossmodality features and the highest cross-modality classification accuracy, while maintaining most of the same modality accuracy.[11]

R.Dharshini and S.Hemanandhini (2016) The important approach in the brain tumor segmentation is to identify the various stages includes benign, malignant and the normal. For 
the process of classifying voxels, a classifier called Self Organizing Map(SOM) is used. Self-Organizing Map (SOM) which includes the unsupervised learning algorithm and Learning Vector Quantization (LVQ) with high diversity data like tumour appearance and its contour deformation. On comparing with the other approaches, instead of using Multimodal MRI images for clustering of voxels SOM has been used. A feature vector has been constructed in which the features for the constructing vector have been gained from the Discrete Wavelet Transform (DWT) coefficients. This has been constructed mainly for identifying tissue types which includes White Matter (WM), Grey Matter (GM), Cerebrospinal Fluid (CSF) and sometimes pathological tissues. The accuracies and the performance are provided in terms of training performance and the classification accuracies.[12]

Anupurba Nandi, (2015) This paper deals with detection of brain tumour from MR images of the brain. The brain is the anterior most part of the nervous system. Tumour is a rapid uncontrolled growth of cells. Magnetic Resonance Imaging (MRI) is the device required to diagnose brain tumour. The normal MR images are not that suitable for fine analysis, so segmentation is an important process required for efficiently analyzing the tumour images. Clustering is suitable for biomedical image segmentation as it uses unsupervised learning. This paper work uses K-Means clustering where the detected tumour shows some abnormality which is then rectified by the use of morphological operators along with basic image processing techniques to meet the goal of separating the tumour cells from the normal cells.[13]

Ivan Cabria (2015) In this paper we view the intensity of a pixel as equal to its "workload" and employ an unsupervised learning algorithm called potential-K-means that generates a balanced distribution of the pixels into clusters of approximately equal total intensity. The algorithm is based on an analogy with the gravitational force exerted by masses. This balancing requirement introduces a search bias that tends

to generate either small clusters of higher intensity pixels, which overlap with the tumor area, or large clusters of lower intensity pixels. We evaluate the proposed algorithm on the publicly available brain tumor image segmentation (BRATS) MRI benchmark by comparing the center of the cluster that overlaps with the tumor, with the center of the tumor in the corresponding ground truth segmentation. We compare the proposed algorithm with the well-known K-means and with the Force clustering algorithm by Kalantari et al. (2009), which follows a different Physics analogy, but it is also based on a balancing criteria. Experimental results show that K-means is not suitable for tumor localization and that potential-K-mean and Kalantari's approach are comparable. However, the performance of Kalantari's approach is highly dependent on a parameter whose value needs to be set a priori, but without an informed way of doing so, which makes the present proposed method more practical.[14]

Ayşe Demirhan, et. Al.(2015)In this study, we present a new tissue segmentation algorithm that segments brain MR images into tumor, edema, white matter (WM), gray matter (GM) and cerebrospinal fluid (CSF). The detection of the healthy tissues is performed simultaneously with the diseased tissues because examining the change caused by the spread of tumor and edema on healthy tissues is very important for treatment planning. We used T1, T2 and FLAIR MR images of 20 subjects suffering from glial tumor. We developed an algorithm for stripping the skull before the segmentation process. The segmentation is performed using self-organizing map (SOM) that is trained with unsupervised learning algorithm and finetuned with learning vector quantization (LVQ). Unlike other studies, we developed an algorithm for clustering the SOM instead of using an additional network. Input feature vector is constructed with the features obtained from stationary wavelet transform (SWT) coefficients. The results showed that average Dice similarity indexes are $91 \%$ for WM, $87 \%$ for GM, 96\% for CSF, $61 \%$ for tumor, and $77 \%$ for edema.[15]

Chen-Ping $\mathrm{Yu}$, et. Al.(2014) Automatic detection and segmentation of brain tumors in 3D MR neuroimages can significantly aid early diagnosis, surgical planning, and followup assessment. However, due to diverse location and varying size, primary and metastatic tumors present substantial challenges for detection. We present a fully automatic, unsupervised algorithm that can detect single and multiple tumors from 3 to $28,079 \mathrm{~mm} 3$ in volume. Using 20 clinical 3D MR scans containing from 1 to 15 tumors per scan, the proposed approach achieves between $87.84 \%$ and $95.30 \%$ detection rate and an average end-to-end running time of under 3 minutes. In addition, 5 normal clinical 3D MR scans are evaluated quantitatively to demonstrate that the approach has the potential to discriminate between abnormal and normal brains.[16]

\section{PROPOSE WORK \\ 4.1 Problem Statement}

Difficult to predict the number of clusters (K-Value).Initial seeds have a strong impact on the final results. The order of the data has an impact on the final results. Sensitive to scale: rescaling your datasets (normalization or standardization) will completely change results. While this itself is not bad, not realizing that you have to spend extra on to scaling your data might be bad.

\subsection{Propose Methodology}

In our propose work MRI Brain Tumor Segmentation method based on K-means (KM) Clustering Algorithm, Fuzzy C-means (FCM) Clustering and Adaptive regularized kernel based fuzzy clustering means (ARKFCM). First take an original image and Existing, Adaptive Regularized Kernel based Fuzzy Clustering Means (ARKFCM) is implemented for extracting the nuclei and non-nuclei images of (Histopathological ROI image). In ARKFCM, the result occurred in two sets of images, both the images are examined for generating the clustering cells. the combined ARKFCM image contains several overlapping regions of cells. Here, the overlapping states (combination of nuclei and non-nuclei cells), is essential to separate those cells to determine the affected cells of liver cirrhosis.

\subsection{Propose Algorithm}

1. start

2. Browse original image from dataset.

3. Resize original image (256 256).

4. Convert image from RGB to gray.

5. Apply k means clustering on this gray scale image.

6. Apply fuzzy c means clustering.

7. Result of k means clustering.

8. Result of fuzzy c means.

9. Use ARKFCM clustering.

10. Results of ARKFCM clustering

11. Exit. 


\subsection{Flowchart}

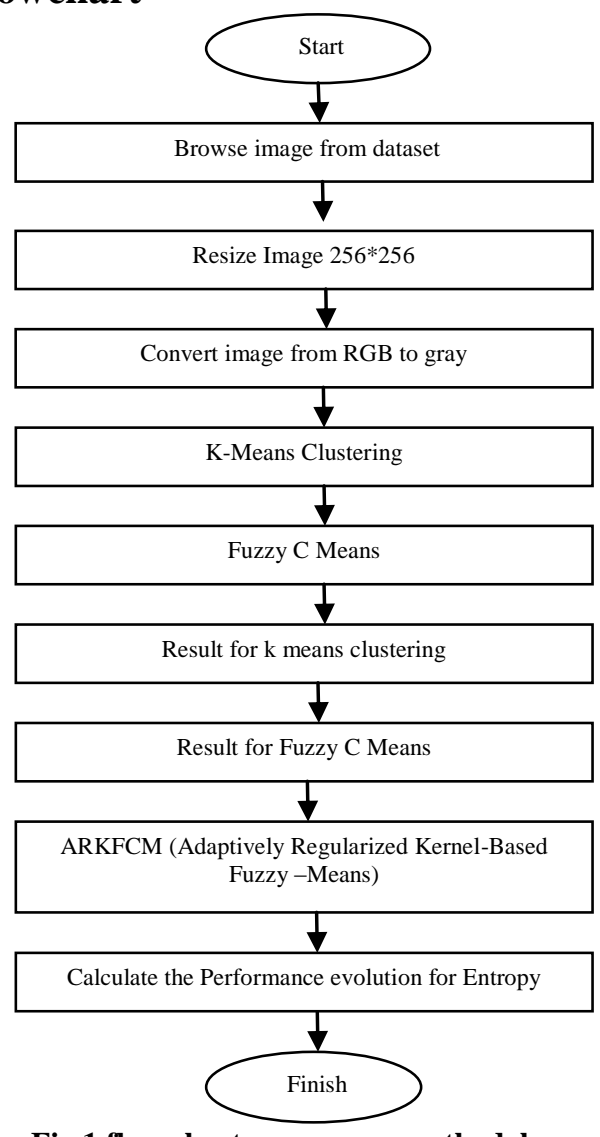

Fig.1.flow chart on propose methodology

\section{EXPERIMENTAL RESULT ANALYSIS}

The experimental analysis use MRI Brain Tumor Segmentation pictures for performance evaluation. To quantitatively compare the results of the above mentioned methods, we apply -means (KM) Clustering Algorithm, Fuzzy C-means (FCM) and the Adaptive regularized kernel based fuzzy clustering means (ARKFCM) is applied to contains several overlapping regions of cells. The algorithm is designed on MATLABR17 using Image Processing toolbox.

First we run this code and we obtained this type of menu bar :

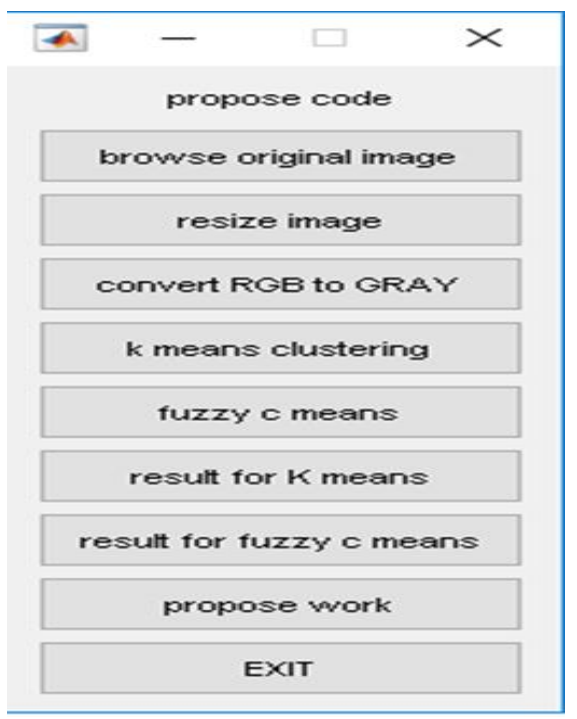

Fig.2.In this menu bar there are 6 steps

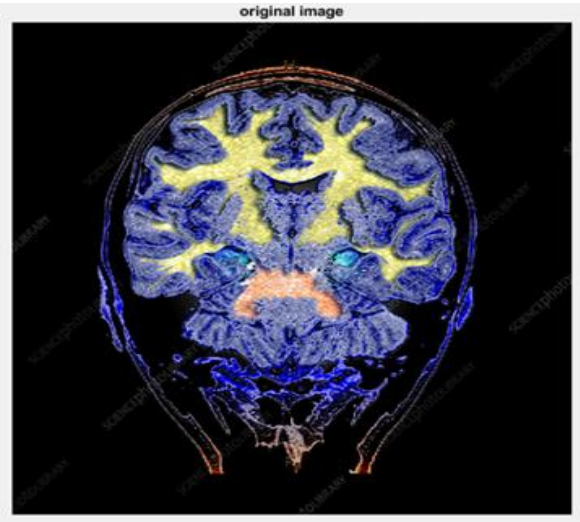

Fig.3.First we browse original image from dataset

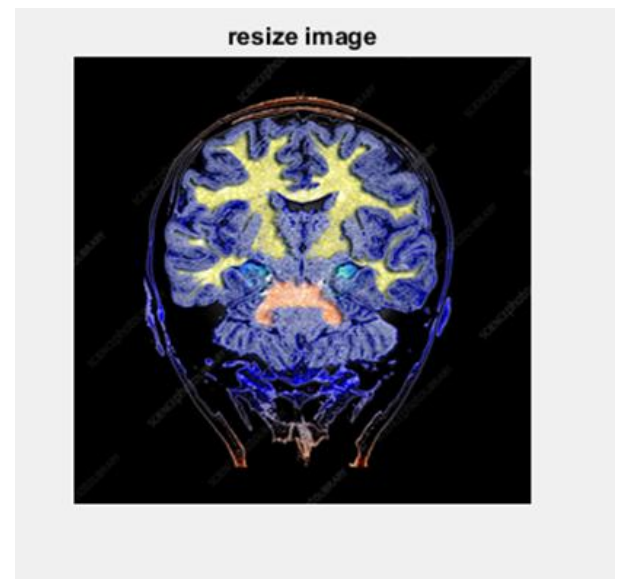

Fig.4.Resize image (256 256)

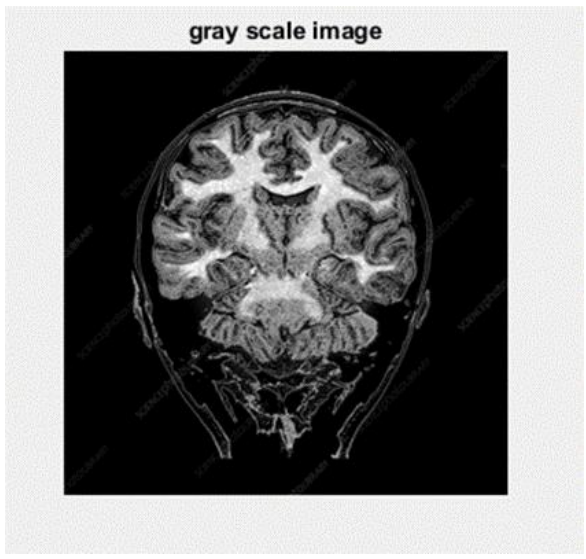

Fig.5.Convert this image from RGB to Gray 


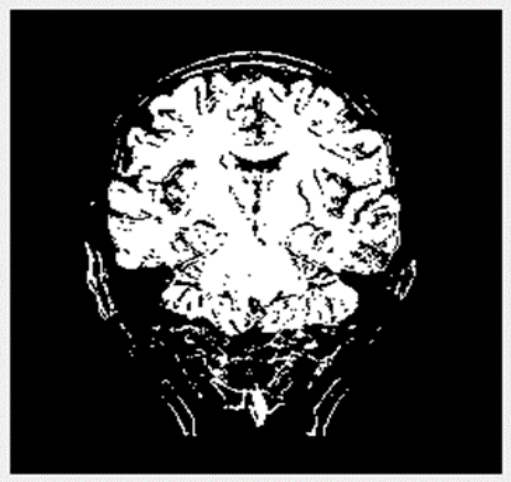

Fig.6.K means clustering

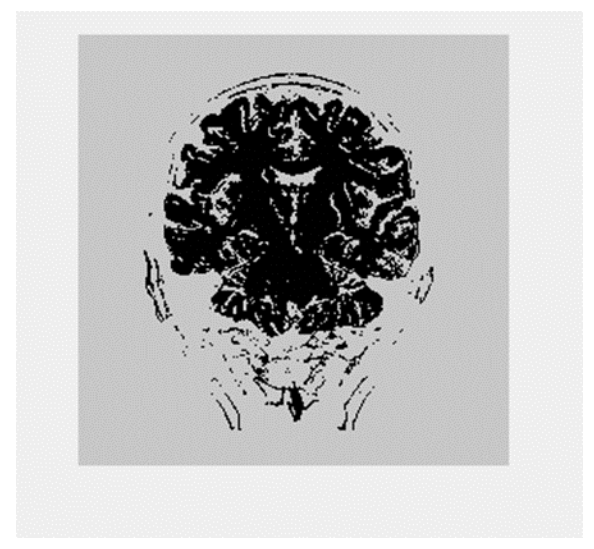

Fig.7.Fuzzy c means

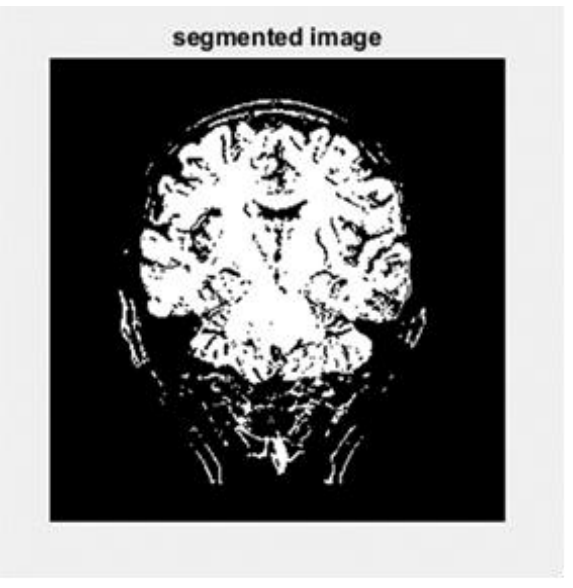

Fig.8.Propose work

Table 1. Comparison on Base PSNR and Propose PSNR for different method

\begin{tabular}{|c|c|c|c|}
\hline I.name & $\begin{array}{c}\text { (BASE)PSNR } \\
\text { K MEANS }\end{array}$ & $\begin{array}{c}\text { (BASE)PSNR } \\
\text { FCM }\end{array}$ & $\begin{array}{c}\text { (PROPOSE)PSNR } \\
\text { ARKFCM }\end{array}$ \\
\hline 3.jpg & 60.5332 & 61.0623 & 80.2753 \\
\hline 4.jpg & 58.0867 & 58.3520 & 79.5493 \\
& & & \\
\hline
\end{tabular}

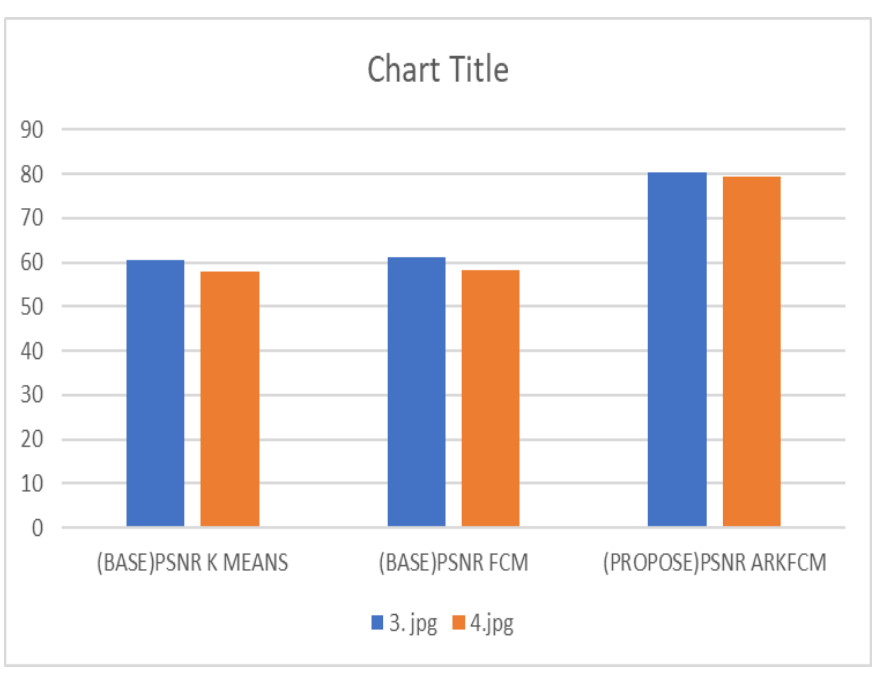

Fig.9 Comparison graph on Base PSNR and Propose PSNR for different metho

Table 2. Comparison on Base MSE and Propose MSE for different method

\begin{tabular}{|c|c|c|c|}
\hline I.name & $\begin{array}{c}\text { (BASE)MSE } \\
\text { K MEANS }\end{array}$ & $\begin{array}{c}\text { (BASE)MSE } \\
\text { FCM }\end{array}$ & $\begin{array}{c}\text { (PROPOSE)MSE } \\
\text { ARKFCM }\end{array}$ \\
\hline 3.jpg & 0.1329 & 0.1289 & 0.0839 \\
\hline 4.jpg & 0.1356 & 0.1337 & 0.0834 \\
& & & \\
\hline
\end{tabular}

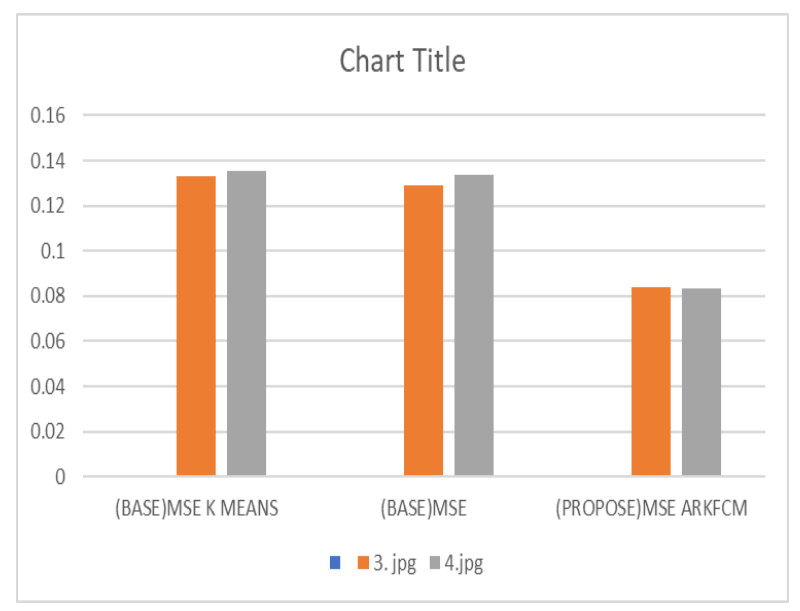

Fig.10 Comparison graph on Base MSE and Propose MSE for different method

\section{CONCLUSION}

An adaptively regularized kernel-based FCM framework has been proposed to enhance the original FCM for higher segmentation low computational cost. An adaptively regularized kernel-based fuzzy $\square$-means clustering framework is proposed for segmentation of brain magnetic resonance images. In this paper apply adaptively Regularized Kernel-Based Fuzzy CMeans Clustering method for improves image result However, the problem of image segmentation speed is still an important problem in image processing. Therefore, how to improve the 
segmentation speed of different algorithms is an indispensable topic. In our future work, we will focus on the image segmentation.

\section{REFERENCES}

[1] G. Li, L. Bai, C. Zhu, E. Wu and R. Ma, "A Novel Method of Synthetic CT Generation from MR Images Based on Convolutional Neural Networks," 2018 11th International Congress on Image and Signal Processing, BioMedical Engineering and Informatics (CISP-BMEI), Beijing, China, 2018, pp. 1-5.

[2] Z. Guo, X. Li, H. Huang, N. Guo and Q. Li, "Medical image segmentation based on multi-modal convolutional neural network: Study on image fusion schemes," 2018 IEEE 15th International Symposium on Biomedical Imaging (ISBI 2018), Washington, DC, 2018, pp. 903-907.

[3] A. Van Opbroek, H. C. Achterberg, M. W. Vernooij and M. De Bruijne, "Transfer Learning for Image Segmentation by Combining Image Weighting and Kernel Learning," in IEEE Transactions on Medical Imaging, vol. 38, no. 1, pp. 213-224, Jan. 2019.

[4] Sandeep Panda, Sanat Sahu, Pradeep Jena, and Subhagata Chattopadhyay, "Comparing Fuzzy-C Means and K-Means Clustering Techniques: A Comprehensive Study", Springer-Verlag Berlin Heidelberg 2012, D.C. Wyld et al. (Eds.): Advances in Computer Science, Eng. \& Appl., AISC 166, pp. 451-460.

[5] Amirhadi Jahanbakhsh Pourjabari, Mojtaba Seyedzadegan, "An improved method of fuzzy c-means clustering by using feature selection and weighting", IJCSNS International Journal of Computer Science and Network Security, VOL.16 No.10, October 2016,pp.64-69.

[6] B. Srinivas and G. S. Rao, "Unsupervised learning algorithms for MRI brain tumor segmentation," 2018 Conference on Signal Processing And Communication Engineering Systems (SPACES), Vijayawada, 2018, pp. 181-184.

[7] Preeti Panwar, Girdhar Gopal, Rakesh Kumar, "Image Segmentation using K-means clustering and Thresholding", International Research Journal of Engineering and Technology (IRJET) e-ISSN: 2395 -0056 Volume: 03, May-2016,pp.1787-1793.

[8] Nameirakpam Dhanachandra and Yambem Jina Chanu, "Image Segmentation Method using K-means Clustering
Algorithm for Color Image", Advanced Research in Electrical and Electronic Engineering p-ISSN: 2349-5804; e-ISSN: 2349-5812 Volume 2, July-September (2015), pp.68-72.

[9] Sibina N and Vimal Raj, "Image Segmentation Using Fuzzy Based Clustering Algorithm And Its Application For Denoising The Digital Images", International Journal of Emerging Trends in Science and Technology(IJETST),may 2016,pp.681-688.

[10] A. SRUTHI1 , M. RAVI KISHORE, “AR Kernel-Based Fuzzy C Method for Brain Tumour Segmentation of MR Images A. SRUTHI1 , M. RAVI KISHORE”. ISSN 2319 8885 Vol.05,Issue.19 July-2016, Pages:3812-38162016 IJSETR.

[11] Gijs van Tulder and Marleen de Bruijne (2018)., "Learning Cross-Modality Representations from Multi-Modal Images", IEEE Transactions on Medical Imaging,pp.1-11.

[12] R.Dharshini and S.Hemanandhini (2016). "Brain tumor segmentation based on Self Organising Map and Discrete Wavelet Transform", 2016 International Conference on Computer Communication and Informatics (ICCCI), pp.19.

[13] Anupurba Nandi, (2015), "Detection of human brain tumour using MRI image segmentation and morphological operators", 2015 IEEE International Conference on Computer Graphics, Vision and Information Security (CGVIS),pp.55-60.

[14] Ivan Cabria and Iker Gondra (2015), "Automated Localization of Brain Tumors in MRI Using Potential-KMeans Clustering Algorithm" 2015 12th Conference on Computer and Robot Vision, pp.125-132.

[15] Ayşe Demirhan, Mustafa Törü, and İnan Güler (2015), "Segmentation of Tumor and Edema Along With Healthy Tissues of Brain Using Wavelets and Neural Networks", IEEE Journal of Biomedical and Health Informatics, pp.17.

[16] Chen-Ping Yu, Guilherme Ruppert, Robert Collins, Dan Nguyen, Alexandre Falcao, and Yanxi Liu (2014), "3D blob based brain tumor detection and segmentation in MR images", 2014 IEEE 11th International Symposium on Biomedical Imaging (ISBI),pp.1192-1197. 